\title{
On common fixed points that belong to the zero set of a certain function
}

\author{
Erdal Karapınar ${ }^{\mathrm{a}}$, Bessem Samet ${ }^{\mathrm{b}, *}$, Priya Shahi \\ ${ }^{a}$ Department of Mathematics, Atilim University, Incek, Ankara, 06836, Turkey. \\ ${ }^{b}$ Department of Mathematics, College of Science, King Saud University, P.O. Box 2455, Riyadh, 11451, Saudi Arabia. \\ ${ }^{c}$ St. Andrews College of Arts, Science and Commerce, St. Dominic Road, Bandra (West), Mumbai 400 050, India. \\ Communicated by M. Eslamian
}

\begin{abstract}
We provide sufficient conditions under which the set of common fixed points of two self-mappings $f, g: X \rightarrow X$ is nonempty, and every common fixed point of $f$ and $g$ is the zero of a given function $\varphi: X \rightarrow[0, \infty)$. Next, we show the usefulness of our obtained result in partial metric fixed point theory. (C)2017 All rights reserved.
\end{abstract}

Keywords: $\varphi$-admissibility, common fixed point, zero set, partial metric.

2010 MSC: 54H25, 47H10.

\section{Introduction and preliminaries}

Recently, Karapinar et al. [6] studied the following problem: for a given mapping $T: X \rightarrow X$ and a given function $\varphi: X \rightarrow[0, \infty)$, find sufficient conditions for which the set of fixed points of $T$ is nonempty, and every fixed point of $T$ is a zero of the function $\varphi$. In order to solve the above problem, the following concepts were introduced in [6]. Let $\mathrm{F}_{\mathrm{T}}$ be the set of fixed points of $\mathrm{T}$, that is,

$$
\mathrm{F}_{\mathrm{T}}=\{x \in \mathrm{X}: \mathrm{T} x=x\},
$$

and $Z_{\varphi}$ be the set of zeros of $\varphi$, that is,

$$
\mathrm{Z}_{\varphi}=\varphi^{-1}(\{0\}) .
$$

The set $\mathrm{F}_{\mathrm{T}}$ is said to be $\varphi$-admissible iff $\mathrm{F}_{\mathrm{T}} \neq \emptyset$ and $\mathrm{F}_{\mathrm{T}} \subseteq \mathrm{Z}_{\varphi}$.

Let $\mathcal{F}$ be the set of functions $F:[0, \infty)^{3} \rightarrow[0, \infty)$ satisfying the following conditions:

(F1) $\max \{a, b\} \leqslant F(a, b, c)$ for all $a, b, c \geqslant 0$;

(F2) $F(a, 0,0)=a$ for all $a \geqslant 0$;

(F3) $\mathrm{F}$ is continuous.

\footnotetext{
${ }^{*}$ Corresponding author

Email addresses: erdalkarapinar@yahoo.com (Erdal Karapınar), bsamet@ksu.edu.sa (Bessem Samet), priya.thaparian@gmail.com (Priya Shahi)

doi:10.22436/jnsa.010.07.09
} 
For examples of functions $\mathrm{F}:[0, \infty)^{3} \rightarrow[0, \infty)$ that belong to the set $\mathcal{F}$, we refer to [6].

Let $\Psi$ be the set of functions $\psi:[0, \infty) \rightarrow[0, \infty)$ satisfying the following conditions:

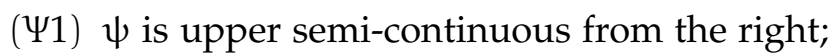

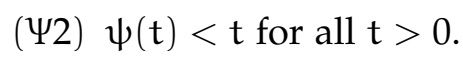

Let $(X, d)$ be a metric space. For given functions $\varphi: X \rightarrow[0, \infty), F \in \mathcal{F}$, and $\psi \in \Psi$, let $\mathcal{T}(\varphi, F, \psi)$ be the class of mappings $T: X \rightarrow X$ satisfying

$$
F(d(T x, T y), \varphi(T x), \varphi(T y)) \leqslant \psi(F(d(x, y), \varphi(x), \varphi(y))), \quad(x, y) \in X \times X .
$$

The following theorem is the main result obtained in [6].

Theorem 1.1. Let $(\mathrm{X}, \mathrm{d})$ be a complete metric space and $\mathrm{T}: \mathrm{X} \rightarrow \mathrm{X}$ be a given operator. Suppose that the following conditions are satisfied:

(i) there exist $\varphi: X \rightarrow[0, \infty), F \in \mathcal{F}$, and $\psi \in \Psi$ such that $T \in \mathcal{T}(\varphi, F, \psi)$;

(ii) $\varphi$ is lower semi-continuous.

Then the set $\mathrm{F}_{\mathrm{T}}$ is $\varphi$-admissible. Moreover, the mapping $\mathrm{T}$ has a unique fixed point.

It was proved in [6] that Theorem 1.1 generalizes several theorems from the literature, including a partial metric version of Boyd-Wong fixed point theorem [3].

Motivated by the contribution [6], in this paper we study the following problem: for given mappings $f, g: X \rightarrow X$ and a given function $\varphi: X \rightarrow[0, \infty)$, find sufficient conditions for which the set of common fixed points of $f$ and $g$ is nonempty, and every common fixed point of $f$ and $g$ is a zero of the function $\varphi$.

Let us denote by $C_{f, g}$ the set of common fixed points of $f, g: X \rightarrow X$, that is,

$$
C_{f, g}=\{x \in X: x=f x=g x\} .
$$

Definition 1.2. Let $f, g: X \rightarrow X$ be two given mappings, and let $\varphi: X \rightarrow[0, \infty)$. We say that $C_{f, g}$ is $\varphi$-admissible iff $\mathrm{C}_{\mathrm{f}, \mathrm{g}} \neq \emptyset$ and $\mathrm{C}_{\mathrm{f}, \mathrm{g}} \subseteq \mathrm{Z}_{\varphi}$.

Let $(X, d)$ be a metric space. For given functions $\varphi: X \rightarrow[0, \infty), F \in \mathcal{F}$, and $\psi \in \Psi$, we denote by $\mathcal{T}_{2}(\varphi, F, \psi)$ the set of pair of mappings $f, g: X \rightarrow X$ satisfying:

$$
F(d(f x, f y), \varphi(f x), \varphi(f y)) \leqslant \psi(F(d(g x, g y), \varphi(g x), \varphi(g y))), \quad(x, y) \in X \times X .
$$

The aim of this paper is to study the $\varphi$-admissibility of the set $C_{f, g}$, where the pair of mappings $f, g: X \rightarrow X$ belongs to the set $\mathcal{T}_{2}(\varphi, F, \psi), F \in \mathcal{F}, \psi \in \Psi$. Next, we show the usefulness of our obtained result in partial metric fixed point theory.

\section{A $\varphi$-admissibility result}

The following theorem, which is the main result in this paper, provides sufficient conditions for the $\varphi$-admissibility of the set $C_{f, g}$.

Theorem 2.1. Let $(\mathrm{X}, \mathrm{d})$ be a complete metric space, $\varphi: \mathrm{X} \rightarrow[0, \infty)$ be a given function, and $\mathrm{f}, \mathrm{g}: \mathrm{X} \rightarrow \mathrm{X}$ be a given pair of mappings. Suppose that

(i) f and g are weakly compatible mappings, that is,

$$
f x=g x, x \in X \Longrightarrow f g x=g f x .
$$


(ii) $f(X) \subseteq g(X)$ and $g(X)$ is closed.

(iii) there exist $F \in \mathcal{F}$ and $\psi \in \Psi$ such that the pair $(f, g) \in \mathcal{T}_{2}(\varphi, F, \psi)$.

(iv) $\varphi$ is lower semi-continuous.

Then the set $\mathrm{C}_{\mathrm{f}, \mathrm{g}}$ is $\varphi$-admissible. Moreover, the mappings $\mathrm{f}$ and $\mathrm{g}$ have a unique common fixed point.

Proof. Let $\xi$ be an arbitrary element of the set $\mathrm{C}_{f, g}$, that is,

$$
\xi \in X, f \xi=g \xi=\xi .
$$

Taking $x=y=\xi$ in (1.1), we obtain

$$
\mathrm{F}(0, \varphi(\xi), \varphi(\xi)) \leqslant \psi(\mathrm{F}(0, \varphi(\xi), \varphi(\xi))) .
$$

If $\psi(F(0, \varphi(\xi), \varphi(\xi))) \neq 0$, from property $(\Psi 2)$, we have

$$
\psi(F(0, \varphi(\xi), \varphi(\xi)))<F(0, \varphi(\xi), \varphi(\xi)),
$$

which yields

$$
\mathrm{F}(0, \varphi(\xi), \varphi(\xi))<\mathrm{F}(0, \varphi(\xi), \varphi(\xi)),
$$

a contradiction. Then $\mathrm{F}(0, \varphi(\xi), \varphi(\xi))=0$, which implies from (F1) that

$$
\varphi(\xi) \leqslant F(0, \varphi(\xi), \varphi(\xi))=0,
$$

and hence, $\xi \in \mathrm{Z}_{\varphi}$. Therefore, we proved that $\mathrm{C}_{f, g} \subseteq \mathrm{Z}_{\varphi}$.

Next, we have to prove that the set $C_{f, g}$ is nonempty. Taking $y_{0}=f x_{0}$, where $x_{0}$ is an arbitrary point in $X$, from (ii), there exists $x_{1} \in X$ such that

$$
y_{0}:=f x_{0}=g x_{1} .
$$

Again, from (ii), there exists $x_{2} \in X$ such that

$$
y_{1}:=f x_{1}=g x_{2} .
$$

Continuing this process, by induction we may construct two sequences $\left\{x_{n}\right\},\left\{y_{n}\right\} \subset X$ defined by

$$
y_{n}=f x_{n}=g x_{n+1}, \quad n \geqslant 0 .
$$

We distinguish two cases.

Case 1. There exists $n_{0} \geqslant 0$ such that $y_{n_{0}}=y_{n_{0}+1}$.

Taking $(x, y)=\left(x_{n_{0}+1}, y_{n_{0}+1}\right)$ in $(1.1)$, we obtain

$$
F\left(d\left(f x_{n_{0}+1}, f y_{n_{0}+1}\right), \varphi\left(f x_{n_{0}+1}\right), \varphi\left(f y_{n_{0}+1}\right)\right) \leqslant \psi\left(F\left(d\left(g x_{n_{0}+1}, g y_{n_{0}+1}\right), \varphi\left(g x_{n_{0}+1}\right), \varphi\left(g y_{n_{0}+1}\right)\right)\right) .
$$

On the other hand, from $y_{n_{0}}=y_{n_{0}+1}$, we have

$$
f x_{n_{0}+1}=y_{n_{0}+1}=y_{n_{0}} \text { and } f y_{n_{0}+1}=f y_{n_{0}} .
$$

Again, from $y_{n_{0}}=y_{n_{0}+1}$, we have

$$
y_{n_{0}}=y_{n_{0}+1}=f x_{n_{0}+1}=g x_{n_{0}+1},
$$

which from (i) yields

$$
g y_{n_{0}+1}=g f x_{n_{0}+1}=f g x_{n_{0}+1}=f y_{n_{0}} .
$$


Hence,

$$
g x_{n_{0}+1}=y_{n_{0}} \text { and } g y_{n_{0}+1}=f y_{n_{0}} .
$$

Substituting (2.2) and (2.3) into (2.1), we obtain

$$
F\left(d\left(y_{n_{0}}, f y_{n_{0}}\right), \varphi\left(y_{n_{0}}\right), \varphi\left(f y_{n_{0}}\right)\right) \leqslant \psi\left(F\left(d\left(y_{n_{0}}, f y_{n_{0}}\right), \varphi\left(y_{n_{0}}\right), \varphi\left(f y_{n_{0}}\right)\right)\right) .
$$

Therefore, if $\mathrm{F}\left(\mathrm{d}\left(\mathrm{y}_{\mathrm{n}_{0}}, f \mathrm{y}_{\mathrm{n}_{0}}\right), \varphi\left(\mathrm{y}_{\mathrm{n}_{0}}\right), \varphi\left(f \mathrm{y}_{\mathrm{n}_{0}}\right)\right) \neq 0$, from property $(\Psi 2)$, we obtain

$$
F\left(d\left(y_{n_{0}}, f y_{n_{0}}\right), \varphi\left(y_{n_{0}}\right), \varphi\left(f y_{n_{0}}\right)\right)<F\left(d\left(y_{n_{0}}, f y_{n_{0}}\right), \varphi\left(y_{n_{0}}\right), \varphi\left(f y_{n_{0}}\right)\right),
$$

which is a contradiction. Hence, we have

$$
F\left(d\left(y_{n_{0}}, f y_{n_{0}}\right), \varphi\left(y_{n_{0}}\right), \varphi\left(f y_{n_{0}}\right)\right)=0,
$$

which implies from (F1) that

$$
d\left(y_{n_{0}}, f y_{n_{0}}\right)=0
$$

that is,

$$
y_{n_{0}}=f y_{n_{0}}=g y_{n_{0}} .
$$

Then we have $\mathrm{y}_{\mathrm{n}_{0}} \in \mathrm{C}_{\mathrm{f}, \mathrm{g}}$.

Case 2. $y_{n} \neq y_{n+1}$, for every $n \geqslant 0$.

In view of (1.1), for all $n \geqslant 1$,

$$
F\left(d\left(f x_{n+1}, f x_{n}\right), \varphi\left(f x_{n+1}\right), \varphi\left(f x_{n}\right)\right) \leqslant \psi\left(F\left(d\left(g x_{n+1}, g x_{n}\right), \varphi\left(g x_{n+1}\right), \varphi\left(g x_{n}\right)\right)\right),
$$

that is,

$$
F\left(d\left(y_{n+1}, y_{n}\right), \varphi\left(y_{n+1}\right), \varphi\left(y_{n}\right)\right) \leqslant \psi\left(F\left(d\left(y_{n}, y_{n-1}\right), \varphi\left(y_{n}\right), \varphi\left(y_{n-1}\right)\right)\right), \quad n \geqslant 1 .
$$

If for some $N \geqslant 1$, we have

$$
F\left(d\left(y_{N}, y_{N-1}\right), \varphi\left(y_{N}\right), \varphi\left(y_{N-1}\right)\right)=0,
$$

then in view of property $(F 1)$, we get $d\left(y_{N}, y_{N-1}\right)=0$, that is, $y_{N}=y_{N-1}$, which is a contradiction with the fact that $y_{n} \neq y_{n+1}$ for every $n \geqslant 0$. Therefore,

$$
F\left(d\left(y_{n}, y_{n-1}\right), \varphi\left(y_{n}\right), \varphi\left(y_{n-1}\right)\right)>0, \quad n \geqslant 1 .
$$

Hence, by property $(\Psi 2)$, we obtain

$$
F\left(d\left(y_{n+1}, y_{n}\right), \varphi\left(y_{n+1}\right), \varphi\left(y_{n}\right)\right)<F\left(d\left(y_{n}, y_{n-1}\right), \varphi\left(y_{n}\right), \varphi\left(y_{n-1}\right)\right), \quad n \geqslant 1 .
$$

As consequence, there exists some $c \geqslant 0$ such that

$$
\lim _{n \rightarrow \infty} F\left(d\left(y_{n}, y_{n-1}\right), \varphi\left(y_{n}\right), \varphi\left(y_{n-1}\right)\right)=c^{+} .
$$

Let us suppose that $c>0$. Therefore, passing to the lim sup as $n \rightarrow \infty$ in (2.4) and using properties ( $\Psi 1)$ and $(\Psi 2)$, we obtain

$$
c \leqslant \limsup _{n \rightarrow \infty} \psi\left(F\left(d\left(y_{n}, y_{n-1}\right), \varphi\left(y_{n}\right), \varphi\left(y_{n-1}\right)\right)\right) \leqslant \psi(c)<c,
$$

which is a contradiction. Then, we deduce that $c=0$, that is,

$$
\lim _{n \rightarrow \infty} F\left(d\left(y_{n}, y_{n-1}\right), \varphi\left(y_{n}\right), \varphi\left(y_{n-1}\right)\right)=0,
$$

which yields from (F1) that

$$
\lim _{n \rightarrow \infty} d\left(y_{n-1}, y_{n}\right)=\lim _{n \rightarrow \infty} \varphi\left(y_{n}\right)=0 .
$$

Now, we shall prove that $\left\{y_{n}\right\}$ is a Cauchy sequence in the metric space $(X, d)$. Suppose that it is not the 
case. Then, there exists $\varepsilon>0$ for which we can find two sequences of positive integers $\{m(k)\}$ and $\{n(k)\}$ such that for all $k \geqslant 0$,

$$
n(k)>m(k)>k, d\left(y_{m}(k), y_{n}(k)\right) \geqslant \varepsilon, d\left(y_{m}(k), y_{n}(k)-1\right)<\varepsilon .
$$

Using (2.6), for all $k \geqslant 0$, we have

$$
\varepsilon \leqslant d\left(y_{m(k)}, y_{n(k)}\right) \leqslant d\left(y_{m(k)}, y_{n(k)-1}\right)+d\left(y_{n(k)-1}, y_{n(k)}\right)<\varepsilon+d\left(y_{n(k)-1}, y_{n}(k)\right),
$$

which yields

$$
\varepsilon \leqslant d\left(y_{m(k)}, y_{n(k)}\right)<\varepsilon+d\left(y_{n(k)-1}, y_{n(k)}\right), \quad k \geqslant 0 .
$$

Passing to the limit as $k \rightarrow \infty$ and using (2.5), we obtain

$$
\lim _{k \rightarrow \infty} d\left(y_{m(k)}, y_{n(k)}\right)=\varepsilon^{+} .
$$

From (2.5) and (2.7), and in view of properties (F1), (F2), and (F3), we get

$$
\lim _{k \rightarrow \infty} F\left(d\left(y_{n(k)}, y_{m(k)}\right), \varphi\left(y_{n(k)}\right), \varphi\left(y_{m(k)}\right)\right)=F(\varepsilon, 0,0)=\varepsilon^{+} .
$$

Hence, by property ( $\Psi 1$, we obtain

$$
\limsup _{k \rightarrow \infty} \psi\left(F\left(d\left(y_{n(k)}, y_{m(k)}\right), \varphi\left(y_{n(k)}\right), \varphi\left(y_{m(k)}\right)\right)\right) \leqslant \psi(\varepsilon) .
$$

On the other hand, using (1.1) and (F1), for all $k \geqslant 0$, we have

$$
\begin{aligned}
\varepsilon & \leqslant d\left(y_{n(k)}, y_{m(k)}\right) \\
& \leqslant d\left(y_{n(k)}, y_{n(k)+1}\right)+d\left(y_{n}(k)+1, y_{m(k)+1}\right)+d\left(y_{m(k)+1}, y_{m(k)}\right) \\
& =d\left(y_{n(k)}, y_{n(k)+1}\right)+d\left(f x_{n(k)+1}, f x_{m(k)+1}\right)+d\left(y_{m}(k)+1, y_{m(k)}\right) \\
& \leqslant d\left(y_{n(k)}, y_{n(k)+1}\right)+F\left(d\left(f x_{n(k)+1}, f x_{m(k)+1}\right), \varphi\left(f x_{n(k)+1}\right), \varphi\left(f x_{m(k)+1}\right)\right)+d\left(y_{m}(k)+1, y_{m(k)}\right) \\
& \leqslant d\left(y_{n(k)}, y_{n(k)+1}\right)+\psi\left(F\left(d\left(g x_{n(k)+1}, g x_{m(k)+1}\right), \varphi\left(g x_{n(k)+1}\right), \varphi\left(g x_{m(k)+1}\right)\right)+d\left(y_{m}(k)+1, y_{m(k)}\right)\right. \\
& =d\left(y_{n(k)}, y_{n(k)+1}\right)+\psi\left(F\left(d\left(y_{n(k)}, y_{m(k)}\right), \varphi\left(y_{n(k)}\right), \varphi\left(y_{m(k)}\right)\right)+d\left(y_{m(k)+1}, y_{m(k)}\right),\right.
\end{aligned}
$$

which yields

$$
\varepsilon \leqslant d\left(y_{n(k)}, y_{n(k)+1}\right)+\psi\left(F\left(d\left(y_{n}(k), y_{m(k)}\right), \varphi\left(y_{n(k)}\right), \varphi\left(y_{m(k)}\right)\right)\right)+d\left(y_{m(k)+1}, y_{m}(k)\right)
$$

for all $k \geqslant 0$. Passing to lim sup as $k \rightarrow \infty$, using (2.5), (2.8), and property ( $\Psi 2$ ), we obtain

$$
\varepsilon \leqslant \psi(\varepsilon)<\varepsilon,
$$

which is a contradiction. As consequence, we deduce that $\left\{y_{n}\right\}$ is a Cauchy sequence in the metric space $(X, d)$.

Due to the fact that $(X, d)$ is a complete metric space, there is some $z \in X$ such that

$$
\lim _{n \rightarrow \infty} y_{n}=\lim _{n \rightarrow \infty} g x_{n}=\lim _{n \rightarrow \infty} f x_{n}=z .
$$

Now, from lower semi-continuity of $\varphi$, and using (2.5), we obtain

$$
0 \leqslant \varphi(z) \leqslant \liminf _{n \rightarrow \infty} \varphi\left(y_{n}\right)=0,
$$

which implies that

$$
z \in \mathbf{Z}_{\varphi}
$$


On the other hand, Since $g(X)$ is a closed set, there exists some $p \in X$ such that $z=g p$. Then, In view of (1.1) and (2.10), we obtain

$$
F\left(d\left(f p, y_{n+1}\right), \varphi(f p), \varphi\left(y_{n+1}\right)\right) \leqslant \psi\left(F\left(d\left(z, y_{n}\right), 0, \varphi\left(y_{n}\right)\right)\right), \quad n \geqslant 1,
$$

which yields from $(\mathrm{F} 1)$

$$
d\left(f p, y_{n+1}\right) \leqslant \psi\left(F\left(d\left(z, y_{n}\right), 0, \varphi\left(y_{n}\right)\right)\right), \quad n \geqslant 1 .
$$

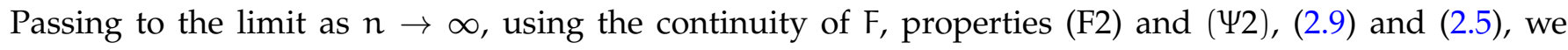
obtain

$$
d(f p, z)=\lim _{n \rightarrow \infty} d\left(f p, y_{n+1}\right)=0,
$$

which yields

$$
z=\mathrm{fp}=\mathrm{gp} .
$$

Now, we shall prove that $z$ is a common fixed point of $f$ and $g$. Since $f$ and $g$ are weakly compatible, therefore, $f g p=g f p$, i.e.,

$$
f z=g z
$$

From (1.1), we have

$$
\mathrm{F}(\mathrm{d}(\mathrm{f} z, \mathrm{fp}), \varphi(\mathrm{f} z), \varphi(\mathrm{fp})) \leqslant \psi(\mathrm{F}(\mathrm{d}(\mathrm{gz}, \mathrm{gp}), \varphi(\mathrm{gz}), \varphi(\mathrm{gp})))
$$

that is,

$$
\mathrm{F}(\mathrm{d}(\mathrm{f} z, z), \varphi(\mathrm{f} z), \varphi(z)) \leqslant \psi(\mathrm{F}(\mathrm{d}(\mathrm{f} z, z), \varphi(\mathrm{f} z), \varphi(z))) .
$$

Therefore, $F(d(f z, z), \varphi(f z), \varphi(z))=0$. Otherwise, from property $(\Psi 2)$, we obtain $F(d(f z, z), \varphi(f z), \varphi(z))<$ $F(d(f z, z), \varphi(f z), \varphi(z))$, which is a contradiction. Hence, by property (F1), we get $d(f z, z)=0$, that is,

$$
z=\mathrm{f} z \text {. }
$$

Next, it follows from (2.11) and (2.12) that

$$
z=\mathrm{f} z=\mathrm{g} z
$$

which yields $z \in C_{f, g}$. As consequence, we deduce that the set $C_{f, g}$ is $\varphi$-admissible.

Finally, we have to prove that $z$ is the unique common fixed point of $f$ and $g$. Let us assume that $w \in \mathrm{C}_{\mathrm{f}, \mathrm{g}}$, that is,

$$
w=f w=g w .
$$

Since the set $C_{f, g}$ is $\varphi$-admissible, we have $z, w \in Z_{\varphi}$, that is,

$$
\varphi(z)=\varphi(w)=0 .
$$

Now, taking $(x, y)=(z, w)$ in $(1.1)$, we obtain

$$
\mathrm{F}(\mathrm{d}(\mathrm{f} z, \mathrm{f} w), \varphi(\mathrm{f} z), \varphi(\mathrm{f} w)) \leqslant \psi(\mathrm{F}(\mathrm{d}(\mathrm{g} z, \mathrm{~g} w), \varphi(\mathrm{g} z), \varphi(\mathrm{g} w))),
$$

that is,

$$
\mathrm{F}(\mathrm{d}(z, w), 0,0) \leqslant \psi(\mathrm{F}(\mathrm{d}(z, w), 0,0)),
$$

which yields $\mathrm{F}(\mathrm{d}(z, w), 0,0)=0$. Otherwise, from property ( $\Psi 2)$, we obtain

$$
\mathrm{F}(\mathrm{d}(z, w), 0,0)<\mathrm{F}(\mathrm{d}(z, w), 0,0),
$$

which is a contradiction. Therefore, by property $(\mathrm{F} 1)$, we obtain $\mathrm{d}(z, w)=0$, that is, $z=w$. As consequence, we deduce that $z \in X$ is the unique common fixed point of $f$ and $g$.

We present the following example in order to illustrate the result given by Theorem 2.1. 
Example 2.2. We endow the finite set $X=\{0,1,2\}$ with the standard metric

$$
d(x, y)=|x-y|, \quad(x, y) \in X \times X .
$$

Let $f, g: X \rightarrow X$ be the mappings defined by

$$
f=\left(\begin{array}{lll}
0 & 1 & 2 \\
0 & 1 & 0
\end{array}\right), g=\left(\begin{array}{lll}
0 & 1 & 2 \\
0 & 2 & 1
\end{array}\right) .
$$

We observe easily that the mappings $f$ and $g$ satisfy conditions (i) and (ii) of Theorem 2.1. Let $\varphi: X \rightarrow$ $[0, \infty)$ be the function defined by

$$
\varphi(x)=x^{2}, \quad x \in X .
$$

Let $\mathrm{F}:[0, \infty)^{3} \rightarrow[0, \infty)$ be the function defined by

$$
F(a, b, c)=a+b+c, \quad a, b, c \geqslant 0 .
$$

Let $\psi:[0, \infty) \rightarrow[0, \infty)$ be the function defined by

$$
\psi(t)=\frac{3}{4} t, \quad t \geqslant 0
$$

It can be easily seen that $F \in \mathcal{F}$ and $\psi \in \Psi$.

We claim that the pair of mappings $(f, g) \in \mathcal{T}_{2}\left(\varphi_{n}, F, \psi\right)$, that is,

$$
|f x-f y|+(f x)^{2}+(f y)^{2} \leqslant \frac{3}{4}\left(|g x-g y|+(g x)^{2}+(g y)^{2}\right), \quad(x, y) \in X \times X .
$$

In order to prove our claim, we discuss different cases.

Case 1. $(x, y)=(0,1)$.

In this case, we have

$$
|f x-f y|+(f x)^{2}+(f y)^{2}=|0-1|+0+1=2
$$

and

$$
\frac{3}{4}\left(|g x-g y|+(g x)^{2}+(g y)^{2}\right)=\frac{3}{4}(|0-2|+0+4)=\frac{9}{2} .
$$

Therefore, (2.13) is satisfied.

Case 2. $(x, y)=(0,2)$.

In this case, we have

$$
|f x-f y|+(f x)^{2}+(f y)^{2}=0 .
$$

Therefore, (2.13) is satisfied.

Case 3. $(x, y)=(1,2)$.

In this case, we have

$$
|f x-f y|+(f x)^{2}+(f y)^{2}=|1-0|+1+0=2
$$

and

$$
\frac{3}{4}\left(|g x-g y|+(g x)^{2}+(g y)^{2}\right)=\frac{3}{4}(|2-1|+4+1)=\frac{9}{2} .
$$

Therefore, (2.13) is satisfied. The other cases follow by symmetry. Then $(f, g) \in \mathcal{T}_{2}\left(\varphi_{n}, F, \psi\right)$.

Next, by Theorem 2.1, the set $C_{f, g}$ is $\varphi$-admissible, and the mappings $f$ and $g$ have a unique common fixed point. In this example, we have

$$
\mathrm{C}_{\mathrm{f}, \mathrm{g}}=\{0\}=\mathrm{Z}_{\varphi} .
$$

Remark 2.3. Taking $g=I_{X}$ (the identity mapping) in Theorem 2.1, we obtain the $\varphi$-admissibility result given by Theorem 1.1. 


\section{Applications to partial metric fixed point theory}

The notion of a partial metric space was introduced in 1994 by Matthews [7] as a part of the study of denotational semantics of data-flow networks, showing that Banach contraction principle can be generalized to the partial metric context for applications in program verification. In this section, as an application of Theorem 2.1, we obtain a common fixed point theorem for two mappings satisfying a Boyd-Wong type contraction on a complete partial metric space. The obtained result is not new (see for instance [1]), however our used techniques are different to those in [1]. More precisely, our main idea consists to rewrite Boyd-Wong contraction on a partial metric space in the form of a generalized Boyd-Wong contraction on a metric space. Next, by applying Theorem 2.1, we deduce the existence and uniqueness of a common fixed point. Our strategy can be used also for different types of contractions defined on a partial metric space.

We suppose that the reader is familiarized with the basic concepts of partial metric spaces and we address him to the classic literature to clarify the doubts, in particular the papers [1, 2, 4, 5, 7-12]. We just recall the following result, which is the main key of our approach.

Lemma $3.1([6])$. Let $(X, p)$ be a partial metric space. Let $d_{p}: X \times X \rightarrow[0, \infty)$ be the mapping defined by

$$
d_{p}(x, y)=2 p(x, y)-p(x, x)-p(y, y), \quad(x, y) \in X \times X .
$$

Then

(i) $\mathrm{d}_{\mathfrak{p}}$ is a metric on $\mathrm{X}$;

(ii) $(X, p)$ is a complete partial metric space if and only if $\left(X, d_{p}\right)$ is a complete metric space;

(iii) the function $X \ni \mathrm{x} \mapsto \mathrm{p}(\mathrm{x}, \mathrm{x})$ is continuous on $\mathrm{X}$ with respect to the topology induced by the metric $\mathrm{d}_{\mathrm{p}}$;

(iv) A subset $\mathrm{M} \subseteq \mathrm{X}$ is closed with respect to the topology induced by the partial metric $\mathrm{p}$ if and only if it is closed with respect to the topology induced by the metric $\mathrm{d}_{\mathrm{p}}$.

Let $(X, p)$ be a partial metric space. For a given function $\psi \in \Psi$, let $\mathcal{T}_{2}(\psi)$ be the set of pair of mappings $f, g: X \rightarrow X$ satisfying the following Boyd-Wong type contraction

$$
p(f x, f y) \leqslant \psi(p(g x, g y)), \quad(x, y) \in X \times X .
$$

We have the following observation.

Lemma 3.2. There exist $\mathrm{F} \in \mathcal{F}$ and $\varphi: \mathrm{X} \rightarrow[0, \infty)$ such that

$$
\mathcal{T}_{2}(\psi) \subset \mathcal{T}_{2}(\varphi, F, \psi), \quad \psi \in \Psi .
$$

Proof. The proof follows from Lemma 3.1. Indeed, let $f, g: X \rightarrow X$ be such that $(f, g) \in \mathcal{T}_{2}(\psi)$ for a certain function $\psi \in \Psi$. Therefore, (3.2) holds for every $(x, y) \in X \times X$. On the other hand, from (3.1) we have

$$
p(x, y)=\frac{d_{p}(x, y)}{2}+\frac{p(x, x)}{2}+\frac{p(y, y)}{2}, \quad(x, y) \in X \times X .
$$

Hence, we obtain

$$
\frac{d_{p}(f x, f y)}{2}+\frac{p(f x, f x)}{2}+\frac{p(f y, f y)}{2} \leqslant \psi\left(\frac{d_{p}(g x, g y)}{2}+\frac{p(g x, g x)}{2}+\frac{p(g y, g y)}{2}\right)
$$

for every $(x, y) \in X \times X$. Next, let us define the functions $F:[0, \infty)^{3} \rightarrow[0, \infty)$ and $\varphi: X \rightarrow[0, \infty)$ by

$$
F(a, b, c)=a+b+c, \quad a, b, c \geqslant 0,
$$

and

$$
\varphi(x)=\frac{p(x, x)}{2}, \quad x \geqslant 0
$$


We obtain

$$
F\left(D_{p}(f x, f y), \varphi(f x), \varphi(f y)\right) \leqslant \psi\left(F\left(D_{p}(g x, g y), \varphi(g x), \varphi(g y)\right)\right), \quad(x, y) \in X \times X,
$$

where $D_{p}$ is the metric on $X$ defined by

$$
D_{p}(x, y)=\frac{d_{p}(x, y)}{2}, \quad(x, y) \in X \times X
$$

Remark 3.3. Note that from Lemma 3.1 (iii), the function $\varphi$ defined by (3.3) is continuous on $X$ with respect to the topology induced by the metric $D_{p}$.

Now, using Lemma 3.1, Lemma 3.2, Remark 3.3, and Theorem 2.1, we deduce immediately the following common fixed point result on a partial metric space.

Corollary 3.4. Let $(\mathrm{X}, \mathrm{p})$ be a complete metric space, and let $\mathrm{f}, \mathrm{g}: \mathrm{X} \rightarrow \mathrm{X}$ be a given pair of mappings. Suppose that

(i) $f$ and $g$ are weakly compatible mappings;

(ii) $f(X) \subseteq g(X)$ and $g(X)$ is closed;

(iii) there exists $\psi \in \Psi$ such that the pair $(f, g) \in \mathcal{T}_{2}(\psi)$.

Then $\mathrm{f}$ and $\mathrm{g}$ have a unique common fixed point $\chi^{*} \in \mathrm{X}$. Moreover, we have $\mathrm{p}\left(\mathrm{x}^{*}, \mathrm{x}^{*}\right)=0$.

\section{Acknowledgment}

The second author extends his appreciation to the Deanship of Scientific Research at King Saud University for funding this work through research group No RGP-237.

\section{References}

[1] M. Abbas, I. Altun, S. Romaguera, Common fixed points of Ćirić-type contractions on partial metric spaces, Publ. Math. Debrecen, 82 (2013), 425-438. 3

[2] T. Abdeljawad, E. Karapınar, K. Taş, A generalized contraction principle with control functions on partial metric spaces, Comput. Math. Appl., 63 (2012), 716-719. 3

[3] D. W. Boyd, J. S. W. Wong, On nonlinear contractions, Proc. Amer. Math. Soc., 20 (1969), 458-464. 1

[4] L. Ćirić, B. Samet, H. Aydi, C. Vetro, Common fixed points of generalized contractions on partial metric spaces and an application, Appl. Math. Comput., 218 (2011), 2398-2406. 3

[5] R. Heckmann, Approximation of metric spaces by partial metric spaces, Applications of ordered sets in computer science, Braunschweig, (1996), Appl. Categ. Structures, 7 (1999), 71-83. 3

[6] E. Karapinar, D. O'Regan, B. Samet, On the existence of fixed points that belong to the zero set of a certain function, Fixed Point Theory Appl., 2015 (2015), 14 pages. 1, 1, 3.1

[7] S. G. Matthews, Partial metric topology, Papers on general topology and applications, Flushing, NY, (1992), Ann. New York Acad. Sci., New York Acad. Sci., New York, 728 (1994), 183-197. 3

[8] S. Oltra, O. Valero, Banach's fixed point theorem for partial metric spaces, Rend. Istit. Mat. Univ. Trieste, 36 (2004), 17-26.

[9] S. Romaguera, A Kirk type characterization of completeness for partial metric spaces, Fixed Point Theory Appl., 2010 (2010), 6 pages.

[10] S. Romaguera, Fixed point theorems for generalized contractions on partial metric spaces, Topology Appl., 218 (2011), 2398-2406.

[11] S. Romaguera, Matkowski's type theorems for generalized contractions on (ordered) partial metric spaces, Appl. Gen. Topol., 12 (2011), 213-220.

[12] O. Valero, On Banach fixed point theorems for partial metric spaces, Appl. Gen. Topol., 6 (2005), 229-240. 3 